\title{
Association between gonadal hormones and osteoporosis in schizophrenia patients with risperidone monotherapy: a cross-sectional study
}

\author{
Yi Chen ${ }^{1}$, Yaoyao Zhang ${ }^{2}$, Kaili Fan ${ }^{2}$, Weiqian Xu ${ }^{3}$, Chao Teng ${ }^{4}$, Shuangshuang Wang ${ }^{5}$, Wei Tang ${ }^{1,2}$, Xiaomin Zhu \\ Corresp. 5 \\ ${ }^{1}$ Department of Psychiatry, The Affiliated Kangning Hospital of Wenzhou Medical University, Wenzhou, Zhejiang, China \\ 2 \\ 2 Department of Psychiatry, Wenzhou Medical University, Wenzhou, Zhejiang, China \\ 3 Department of Psychiatry, The Second People's Hospital of TaiZhou, Taizhou, Zhejiang, China \\ 4 Department of Psychiatry, Zhejiang Chinese Medical University, Hangzhou, Zhejiang, China \\ 5 Department of Psychiatry, Suzhou Guangji Hospital, the Affiliated Guangji Hospital of Soochow University, Suzhou, Jiangsu, China \\ Corresponding Author: Xiaomin Zhu \\ Email address: lizamin@163.com
}

Objective: Patients with schizophrenia are at increased risk of osteoporosis. This study firstly learned the osteoporosis rate in patients with schizophrenia]then explored the association between serum gonadal hormone levels and osteoporosis among these patients.

Methods: A total of 250 patients with schizophrenia and 288 healthy controls were recruited. Osteoporosis was defined by decreased bone mineral density (BMD) of the calcaneus. Serum fasting levels of gonadal hormones (prolactin, estradiol, testosterone, progesterone, follicle-stimulating hormone, luteinizing hormone) were determined. The relationship between osteoporosis and hormone levels was statistically analyzed by binary logistic regression analysis.

Results: Our results showed that patients with schizophrenia had a markedly higher rate of osteoporosis (24.4\% vs. $10.1 \%$ ) compared to healthy controls $(P<0.001)$. Patients with osteoporosis were older, had a longer disease course, and lower body mass index (BMI) compared to patients without osteoporosis (all $P$ $<0.05$ ). Regarding gonadal hormones, we found significantly higher prolactin, but lower estradiol, levels in patients with osteoporosis than those without osteoporosis (both $\mathrm{P}<0.05$ ). The regression analysis found that $\mathrm{PRL}(\mathrm{OR}=1.1,95 \% \mathrm{Cl}=1.08 \sim 1.15, \mathrm{P}<0.001)$ and $\mathrm{E} 2$ level $(\mathrm{OR}=0.9,95 \% \mathrm{Cl}=0.96 \sim 0.99, \mathrm{P}$ $=0.011$ ) were significantly associated with osteoporosis in patients with schizophrenia.

Conclusion: Our results suggest a high rate of osteoporosis in risperidone-treated patients with schizophrenia. Increased prolactin and reduced estradiol are significantly associated with osteoporosis. 
1 Association between gonadal hormones and osteoporosis in

2 schizophrenia patients undergoing risperidone monotherapy: a

3 cross-sectional study

4 Yi Chen ${ }^{1}$, Yaoyao Zhang ${ }^{2}$, Kaili Fan², Weiqian $\mathrm{Xu}^{3}$, Chao Teng ${ }^{4}$, Shuangshuang Wang ${ }^{5}$, Wei

5 Tang ${ }^{1,2}$, Xiaomin $\mathrm{Zhu}^{5, *}$

$6{ }^{1}$ Department of Psychiatry, the Affiliated Kangning Hospital of Wenzhou Medical

7 University, Wenzhou, Zhejiang, China

$8 \quad{ }^{2}$ Department of Psychiatry, Wenzhou Medical University, Wenzhou, Zhejiang, China

9 32Department of Psychiatry, the Second People's Hospital of Taizhou, Taizhou, Zhejiang, China

$10{ }^{4}$ Department of Psychiatry, Zhejiang Chinese Medical University, Hangzhou, Zhejiang, China

$11{ }^{5}$ Department of Psychiatry, Suzhou Guangji Hospital, the Affiliated Guangji Hospital of

12 Soochow University, Suzhou, Jiangsu, China

13

$14 *$ Corresponding authors

15 Xiaomin Zhu, Email: 1izamin@163.com

16 
18 Abstract

19 Objective: Patients with schizophrenia are at increased risk of osteoporosis. This study first

20 determined the osteoporosis rate in patients with schizophrenia and then explored the association

21 between serum gonadal hormone levels and osteoporosis among these patients.

22 Methods: A total of 250 patients with schizophrenia and 288 healthy controls were recruited.

23 Osteoporosis was defined by decreased bone mineral density (BMD) of the calcaneus. Serum

24 fasting levels of gonadal hormones (prolactin, estradiol, testosterone, progesterone, follicle-

25 stimulating hormone, luteinizing hormone) were determined. The relationship between

26 osteoporosis and hormone levels was statistically analyzed by binary logistic regression analysis.

27 Results: Our results showed that patients with schizophrenia had a markedly higher rate of

28 osteoporosis $(24.4 \%$ vs. $10.1 \%)$ than healthy controls $(\mathrm{P}<0.001)$. Patients with osteoporosis were

29 older, had a longer disease course, and had a lower body mass index (BMI) than patients without

30 osteoporosis (all $\mathrm{P}<0.05$ ). Regarding gonadal hormones, we found significantly higher prolactin

31 but lower estradiol levels in patients with osteoporosis than in those without osteoporosis (both P

$32<0.05)$. The regression analysis revealed that PRL $(\mathrm{OR}=1.1,95 \% \mathrm{CI}=1.08 \sim 1.15, \mathrm{P}<0.001)$ and

33 E2 levels $(\mathrm{OR}=0.9,95 \% \mathrm{CI}=0.96 \sim 0.99, \mathrm{P}=0.011)$ were significantly associated with osteoporosis

34 in patients with schizophrenia.

35 Conclusion: Our results indicate that patients with schizophrenia who are being treated with

36 risperidone have a high rate of osteoporosis. Increased prolactin and reduced estradiol levels are

37 significantly associated with osteoporosis.

38 Keywords: schizophrenia; risperidone; gonadal hormone; osteoporosis; bone mineral density. 
44

45

46

47

\section{Introduction}

Osteoporosis is a degenerative disease that is characterized by a decrease in bone mineral density (BMD) and results in an increased risk of fractures ${ }^{1}$. Approximately 200 million people worldwide are affected by osteoporosis, which increases their morbidity and mortality ${ }^{2}$.

Numerous studies have been conducted to explore risk factors for osteoporosis, and commonly reported risk factors are old age, female sex, insufficient calcium intake, inadequate physical activity, excessive smoking, excessive drinking and use of antipsychotics ${ }^{3,4}$.

Schizophrenia is a severe, chronic, and debilitating disorder that affects approximately $1 \%$ of the global population ${ }^{5}$. Antipsychotic drugs are considered the primary treatment for schizophrenia. Although these drugs have significant benefits for psychotic symptoms, they can induce health problems such as metabolic syndrome, cardiovascular diseases, sexual dysfunction, and osteoporosis 6 . Previous studies have demonstrated that patients with schizophrenia have a higher risk of osteoporosis than the general population ${ }^{2,7}$.

The underlying mechanisms of increased osteoporosis risk in patients with schizophrenia are still unclear. However, studies have shown that, in addition to poor nutrition, reduced physical activity, excessive smoking, and drinking, the main reason that patients with schizophrenia develop osteoporosis is the use of antipsychotic drugs ${ }^{3,8}$. Antipsychotics can elevate the secretion of prolactin (PRL) via the dopamine D2 receptor-blocking effect ${ }^{9}$. In addition, hyperprolactinemia caused by antipsychotics can lead to estrogen and androgen deficiencies, which can accelerate bone loss and increase the risk of osteoporosis ${ }^{10}$. A large body of evidence supports that decreased estrogen levels can increase bone resorption by prolonging 
66 the life of osteoclasts ${ }^{11,12}$ and that androgen deficiency can lead to an imbalance of osteoblast

67 and osteoclast activity, resulting in decreased osteogenesis ${ }^{13}$. Taken together, these findings

68

69

suggest that abnormal levels of gonadal hormones due to the use of antipsychotic drugs could be associated with osteoporosis in patients with schizophrenia. To further explore this hypothesis, we focused on schizophrenia patients using a single antipsychotic drug, risperidone, which is commonly used in our clinical practice.

In clinical practice, risperidone is a widely used second-generation antipsychotic (SGA), as well as a prolactin-elevating compound ${ }^{14}$. Early studies of patients treated with risperidone found high PRL levels to be associated with low BMD values ${ }^{15}$. However, other studies failed to replicate this pattern ${ }^{16}$. The inconsistent results may be related to confounding factors, such as age, gender and varying levels of physical activity.

In the present study, we aimed to analyze risk factors associated with osteoporosis in inpatients with schizophrenia receiving risperidone monotherapy. We speculate that abnormal gonadal hormone levels may be associated with the onset of osteoporosis in patients with schizophrenia. We hope that this work can provide clinical data on the osteoporosis rate and its associated risks in patients with schizophrenia, which helps us to take measures to prevent osteoporosis and treat it.

\section{Materials and Methods}

\subsection{Participants}

A total of 250 inpatients with schizophrenia who were hospitalized in Kangning Hospital Affiliated to Wenzhou Medical University from May 2018 to June 2020 were included in our 
87 study. All patients met the following criteria: (1) a diagnosis of schizophrenia according to the

Diagnostic and Statistical Manual of Mental Disorders, Fourth Edition (DSM-IV) ${ }^{17}$; (2) age 1875 years old; (3) Han Chinese ethnicity; and (4) undergoing risperidone monotherapy for at least 6 months. The exclusion criteria were as follows: (1) diagnosis of a psychiatric disorder in addition to schizophrenia or a history of substance abuse/dependence disorder; (2) severe cardiovascular, hepatic, or renal diseases that may affect bone metabolism, such as diabetes or hyperthyroidism; (3) pregnancy or breastfeeding; and (4) a history of bone fracture within one year before enrollment. Since admission to the facility, all patients had followed the same diet and activity schedule, which helps to minimize differences in physical exercise and diet between patients. According to the principles of frequency matching, we selected a group of 288 healthy controls from the physical examination center at our hospital. The control group was comparable to the patient group in terms of age and sex. This study was performed in strict accordance with the Declaration of Helsinki and all other relevant national and international regulations. The study protocol was approved by the Medical Ethics Committee of The Affiliated Kangning Hospital of Wenzhou Medical University (approval number: 20180412001). All participants signed informed consent before the formal study. Written informed consent was obtained from all participants prior to their participation in any procedures related to this study.

\subsection{Assessment of participant characteristics}

Detailed demographic and clinical data were collected via a standardized form that was specifically designed for this study. Weight and height were measured in a standardized manner. Participants were barefoot and stood upright, while height was measured to the nearest millimeter. An electronic scale was used to evaluate weight while wearing light indoor clothing. Body mass index (BMI) was calculated as weight in $\mathrm{kg} / \mathrm{square}$ of height in meters. 
110

111

112

113

114

115

116

117

118

119

120

121

122

123

124

125

126

127

128

129

130

\subsection{Definition of osteoporosis}

$\operatorname{BMD}\left(\mathrm{g} / \mathrm{cm}^{2}\right)$ of the calcaneus was measured by a trained ultrasound technician

blind to our research hypotheses in a separate examination center at the hospital using a 3.01

Sahara Clinical Bone Sonometer (Hologic). Quantitative ultrasound of the calcaneus (QUS)

measurements were performed at the right heel (or left heel, if the right heel was inaccessible)

using a 6 Broadband ultrasound attenuation (BUA; DB/MHz) and speed of sound (SOS; m/s) at least twice on each calcaneum. The T-score of QUS refers to the number of standard deviations

(SD) away from the mean T-score of a database of normal values compiled from a healthy young adult population and was calculated as $(0.67 \times \mathrm{BUA}+0.28 \times \mathrm{SOS})-420$. According to World Health Organization (WHO) criteria (World Health Organization Study Group, 1994), 0steoporosis is defined by BMD.

\subsection{Measurement of serum gonadal hormones}

A 10-ml fasting blood sample was drawn from each patient between 6:00 and 9:00 a.m. and stored in ice-cooled ethylenediaminetetraacetic acid tubes. Serum was separated by centrifugation at $5{ }^{\circ} \mathrm{C}$ and stored at $-20{ }^{\circ} \mathrm{C}$. Gonadal hormones, including PRL, estradiol (E2), testosterone $(\mathrm{T})$, progesterone $(\mathrm{P})$, follicle-stimulating hormone (FSH), and luteinizing hormone (LH), were measured using the chemiluminescence immunoassay kits ARCHITECT and ARCHITECT i2000 (Abbott Japan Co., Chiba, Japan).

\subsection{Statistical analysis}

Statistical analyses were performed using SPSS software version 26.0 (SPSS, Chicago, IL). First, we used independent samples t-tests or the Mann-Whitney $U$ test for continuous variables 
131 and the chi-square test for categorical variables to compare differences between groups. Second,

132 using osteoporosis as the dependent variable and age, BMI, total disease course, estradiol level,

133 prolactin level, FSH level and risperidone doses as independent variables, we performed a binary

134 logistic regression analysis with the "enter" method to identify factors independently associated

135 with osteoporosis in patients with schizophrenia. All tests were two-tailed, and the statistical

136 significance level was set as $\mathrm{P} \leq 0.05$.

\section{3. Results}

\subsection{Demographic characteristics of patients and controls}

The demographic data of the participants are presented in Table 1. For patients with

schizophrenia, the average age was $46.3 \pm 10.6$ years, and the average BMI was $24.5 \pm 4.0 \mathrm{~kg} / \mathrm{m}^{2}$.

141 For healthy controls, the average age was $46.0 \pm 10.8$ years, and the average BMI was $23.4 \pm 3.5$

$142 \mathrm{~kg} / \mathrm{m}^{2}$. There were no significant differences in sex or age between patients and controls (age:

$\mathrm{P}=0.613$; sex: $\mathrm{P}=0.101)$. Patients had a significantly higher $\mathrm{BMI}$ than healthy controls $(\mathrm{P}=0.007)$.

Among patients with schizophrenia, the average disease duration was $21.0 \pm 9.0$ years.

\subsection{Rates of osteoporosis in patients and controls}

The rates of osteoporosis were $24.4 \%(61 / 250)$ for patients with schizophrenia and $10.1 \%$

$148(29 / 288)$ for healthy controls. The patient group had a significantly higher rate of osteoporosis than the control group $(\mathrm{P}<0.001)$. 
152

153

154

155

156

157

between schizophrenia patients with and without osteoporosis

Our results showed that schizophrenia patients with osteoporosis were older than those without osteoporosis $(51.7 \pm 11.6$ vs $44.6 \pm 9.7, Z=-4.6, p<0.001)$. Moreover, patients with osteoporosis had a longer duration of illness $(26.2 \pm 9.3$ vs $19.5 \pm 8.8, Z=-4.9, P<0.001)$ and lower

BMI $(23.1 \pm 3.06$ vs $24.9 \pm 4.1, \mathrm{t}=3.5, \mathrm{P}<0.001)$. There were no significant differences in sex or daily risperidone dose between patients with and without osteoporosis (both $\mathrm{P}>0.05$ ). For gonadal hormone levels, patients with osteoporosis had a significantly higher PRL level $(90.9 \pm 20.5$ vs $44.1 \pm 17.2, Z=-10.6, P<0.001)$, a lower E2 level $(55.3 \pm 24.6$ vs $88.0 \pm 45.3, Z=-$ 5.3, $\mathrm{P}<0.001)$ and a higher FSH level $(7.6 \pm 4.1$ vs $6.6 \pm 4.0, \mathrm{Z}=-2.2, \mathrm{P}=0.026)$ than patients without osteoporosis (Table 2).

3.4 Factors associated with osteoporosis in patients with schizophrenia The binary logistic regression analysis found that PRL levels were positively associated with osteoporosis in patients $(\mathrm{OR}=1.1,95 \% \mathrm{CI}=1.08 \sim 1.15, \mathrm{P}<0.001)$, and $\mathrm{E} 2$ levels were negatively associated with osteoporosis in patients $(\mathrm{OR}=0.9,95 \% \mathrm{CI}=0.96 \sim 0.99, \mathrm{P}=0.011)$ (see Table 3), accounting for $52 \%$ of the variance of osteoporosis in patients.

\section{Discussion}

In the present study, we found that schizophrenia patients undergoing risperidone monotherapy had a higher rate of osteoporosis than healthy controls. We demonstrated that $24.4 \%$ of patients treated with risperidone had osteoporosis, which represents a 2.4 -fold 
173

174

increased risk compared to healthy controls. Of note, a recent meta-analysis reported that osteoporosis is over 2.5 times more common in patients with schizophrenia treated with antipsychotics than in age- and sex-matched controls ${ }^{16}$. In previous studies, the recruited patients used different antipsychotics, which may affect the rate of osteoporosis. When we only used risperidone monotherapy, the results were still consistent with the majority of previous studies ${ }^{2}$, 16,18. To the best of our knowledge, this is the first study exploring the rate of osteoporosis in schizophrenia inpatients with risperidone monotherapy.

Despite some investigations, the precise role of antipsychotics in osteoporosis risk remains unclear. One potential mechanism relates to choice in the hypothalamic-pituitarygonadal axis induced by antipsychotics ${ }^{19}$. The dopamine D2 receptor-blocking effect of antipsychotics could elevate the secretion of PRL, causing hyperprolactinemia ${ }^{20}$. The increased PRL level then leads to attenuation of the bone resorption rate, which consequently lowers the secretion of sex hormones such as E2 and $\mathrm{T}^{2,10}$. In light of awareness that different types of antipsychotics have different effects on PRL, a recent meta-analysis and systematic review demonstrated that patients treated with PRL-raising antipsychotics (typical antipsychotics, risperidone, paliperidone, amisulpride) have a higher risk of BMD loss and osteoporosis than patients receiving PRL-decreasing antipsychotics ${ }^{23,24}$. Risperidone, which has difficulty penetrating the blood-brain barrier, is expected to have longer-lasting D2 antagonistic effects in the pituitary system than in the central nervous system, ultimately leading to prolonged hyperprolactinemia and maximal loss of $\mathrm{BMD}^{25}$. Thus, risperidone may be the most likely to cause hormonal dysfunction, thereby increasing osteoporosis. Hence, clinical practice should be 
194 alert to the use of risperidone.

195 To date, no study has been conducted to explore the relationship between hypothalamic-

196 pituitary-gonadal axis-related hormones and osteoporosis in schizophrenia patients receiving

197 risperidone monotherapy. Our study provides new insights into the relationship between the two

198 and further supports the role of gonadal hormones in the risk of osteoporosis in patients receiving risperidone. Specifically, we found that patients with osteoporosis had significantly higher PRL

levels but lower E2 levels than patients without osteoporosis, which is similar to the findings of

previous studies ${ }^{26}$. Moreover, logistic regression analysis confirmed that PRL and E2 were independent predictive factors associated with osteoporosis after controlling for other known

associated factors. Despite some research demonstrating significant correlations between P, FSH and $\mathrm{TH}^{27,28}$, we did not observe such relationships, which is in line with the majority of

previous studies ${ }^{29,30}$. The association of low E2 levels with osteoporosis is consistent with the

high incidence of osteoporosis in postmenopausal women ${ }^{26,30}$ and supports the view that estrogen is involved in bone metabolism.

In addition, our study showed that schizophrenia patients with osteoporosis were older and

had a longer total disease course than those without osteoporosis. These two risk factors have

210 also been reported previously ${ }^{2,31,32}$. It is well known that the aging process increases bone

211 destruction and decreases bone formation ${ }^{23}$. Patients with schizophrenia with a longer disease course may have received longer treatment with antipsychotics, thus resulting in more profound

213 effects on gonadal hormones. However, our logistic regression analysis showed that age and 214 disease course did not have significant clinical effects on osteoporosis. Rather, the results 
215 suggested that gonadal hormones, namely, PRL and E2, are important to osteoporosis risk.

216 Nevertheless, the relationship between osteoporosis and disease course warrants further

217 verification in prospective and longitudinal studies.

218 Unexpectedly, we found a lower BMI in patients with osteoporosis than in patients without

219 osteoporosis. Previous research has reported a significant positive correlation between BMI and

220 osteoporosis ${ }^{1,34}$. One possible reason for this relationship is that the higher BMI caused by SGA

221 use could counteract BMD loss effects in schizophrenia ${ }^{33}$. Although risperidone showed a slight

222 effect on body weight, in the logistic regression analysis, the difference between groups

223 disappeared. Extensive longitudinal research with larger samples is needed to confirm these

224 relationships.

225

The strength of this study is the relatively large sample of schizophrenia patients receiving risperidone monotherapy. However, there are several limitations worth mentioning. First, the cross-sectional nature of this research provides a limited capacity to identify a causal relationship between gonadal hormones and BMD loss or osteoporosis. Second, since all patients were inpatients recruited from one hospital in Wenzhou, our findings may not be generalizable to other settings and outpatient populations. Third, although all patients received risperidone monotherapy for at least six months, we did not collect detailed information about medication history, which may have effects on gonadal hormones. Fourth, we only recruited patients following a similar diet and physical exercise schedule, which will cause selection bias to a certain extent. Finally, future research with a prospective and longitudinal design is required to evaluate causal relations between gonadal hormones and osteoporosis in first-episode and drug- 
236

237

238

239

240

241

242

243

244

245

246

247

248

249

250

251

252

253

254

255

256

257

naïve patients with schizophrenia.

In summary, our results provide further evidence of the increased rate of osteoporosis in

patients with risperidone monotherapy compared to controls. Patients with osteoporosis tended to be older, have a longer disease course, have a higher BMI, have significantly higher PRL levels, and have lower E2 levels than patients without osteoporosis. Our binary regression logistic analysis showed that PRL and E2 levels were independently associated with osteoporosis. These findings suggest that PRL and E2 levels are related to osteoporosis in patients treated with risperidone. Prospective and longitudinal research is warranted to confirm these findings and investigate the underlying mechanism.

\section{Abbreviations}

BMD : Bone Mineral Density; BMI : Body Mass Index; PRL:Prolactin; E2 : estradiol ; T:

Testosterone; $\mathrm{P}$ : Progesterone ; FSH : follicle-stimulating hormone ; $\mathrm{LH}$ : luteinizing hormone ;

SGA : Second Generation Antipsychotic

\section{Acknowledgment}

The present work was supported by the Natural Science Foundation of Jiangsu Province (BK20180213) and the Wenzhou Basic Medical Science and Technology Project

(Y20190478).We are deeply grateful to all the patients with schizophrenia and healthy controls participating in this study as well as to the psychiatrists for their help in the recruitment and diagnosis of schizophrenic patients. 


\section{Conflicts of Interest}

The authors have no conflicts to disclose.

\section{References}

1. Liang M, Zhang B, Deng L, Xu R, Wu H, Chen J. 2019. Effects of Olanzapine on Bone Mineral Density, Glucose, and Lipid Metabolism in Schizophrenia Patients. International Journal of endocrinology;2019:1312804.

2. Cui J, Liu H, Shao J, Xu DM, Wang Y, Fei Z, Wei J, Lu W, Wang CR, He R, Tan Y, Fan Y, Ning Y, Cassidy RM, Soares JC, Huang X, Zhang XY. 2018. Prevalence, risk factors, and clinical characteristics of osteoporosis in Chinese inpatients with schizophrenia. Schizophrenia Research. 195:488-494.

3. Li G, Thabane L, Papaioannou A, Ioannidis G, Levine MA, Adachi JD. 2017. An overview of osteoporosis and frailty in the elderly. BMC Musculoskeletal Disorders. 26;18(1):46.

4. Crews MP, Howes OD. 2012. Is antipsychotic treatment linked to low bone mineral density and osteoporosis? A review of the evidence and the clinical implications. Human Psychopharmacology. 27(1):15-23.

5. Stępnicki P, Kondej M, Kaczor AA. 2018. Current Concepts and Treatments of Schizophrenia. Molecules (Basel, Switzerland). 23(8).

6. Andrade C. 2016. Cardiometabolic Risks in Schizophrenia and Directions for Intervention, 3: Psychopharmacological Interventions. The Journal Of Clinical Psychiatry. 77(9):e1090-e1094.

7. Stubbs B, Gaughran F, Mitchell AJ, De Hert M, Farmer R, Soundy A, Rosenbaum S, Vancampfort D. 2015. Schizophrenia and the risk of fractures: a systematic review and comparative meta-analysis. General Hospital Psychiatry. 37(2):126-133.

8. Halbreich U, Kinon BJ, Gilmore JA, Kahn LS. 2003. Elevated prolactin levels in patients with schizophrenia: mechanisms and related adverse effects. Psychoneuroendocrinology. 28 Suppl 1:53-67.

9. Peuskens J, Pani L, Detraux J, De Hert M. 2014. The effects of novel and newly approved antipsychotics on serum prolactin levels: a comprehensive review. CNS Drugs.28(5):421-453.

10. Okita K, Kanahara N, Nishimura M, Yoshida T, Yasui-Furukori N, Niitsu T, Yoshida T, Ishikawa M, Kimura H, Nomura F, Iyo M. 2014. Second-generation antipsychotics and bone turnover in schizophrenia. Schizophrenia Research. 157(1-3):137-141.

11. Li W, Zhu HM, Xu HD, Zhang B, Huang SM. 2018. CRNDE impacts the proliferation of osteoclast by estrogen deficiency in postmenopausal osteoporosis. European Review For Medical And Pharmacological Sciences. 22(18):5815-5821.

12. Chopko TC, Lindsley CW. 2018. Classics in Chemical Neuroscience: Risperidone. ACS Chemical Neuroscience, 9(7), 1520-1529.

13. Mohamad NV, Soelaiman IN, Chin KY. 2016. A concise review of testosterone and bone health. Clinical Interventions In Aging;11:1317-1324.

14. Bishop JR, Rubin LH, Reilly JL, Pavuluri MN, Sweeney JA. 2012. Risperidone-associated prolactin elevation and markers of bone turnover during acute treatment. Therapeutic Advances In Psychopharmacology;2(3):95-102.

15. Becker D, Liver O, Mester R, Rapoport M, Weizman A, Weiss M. 2003. Risperidone, but not olanzapine, decreases bone mineral density in female premenopausal schizophrenia patients. The Journal of Clinical Psychiatry. 64(7):761-766. 
16. Stubbs B, De Hert M, Sepehry AA, Correll CU, Mitchell AJ, Soundy A, Detraux J, Vancampfort D. 2014. A meta-analysis of prevalence estimates and moderators of low bone mass in Patients with schizophrenia. Acta Psychiatrica Scandinavica. 130(6):470-486.

17. American Psychiatric Association. Diagnostic and statistical manual of mental disorders. 4th ed. Washington, DC: American Psychiatric Association; 1994.

18. Gomez L, Stubbs B, Shirazi A, Vancampfort D, Gaughran F, Lally J. 2016. Lower Bone Mineral Density at the Hip and Lumbar Spine in People with Psychosis Versus Controls: a Comprehensive Review and Skeletal Site-Specific Meta-analysis. Current Osteoporosis Reports. 14(6):249-259.

19. Kishimoto T, Watanabe K, Shimada N, Makita K, Yagi G, Kashima H. 2008. Antipsychotic-induced hyperprolactinemia inhibits the hypothalamo-pituitary-gonadal axis and reduces bone mineral density in male patients with schizophrenia. The Journal Of Clinical Psychiatry. 69(3):385-391.

20. Meaney AM, Smith S, Howes OD, O'Brien M, Murray RM, O'Keane V. 2004. Effects of long-term prolactinraising antipsychotic medication on bone mineral density in patients with schizophrenia. The British Journal Of Psychiatry: The Journal Of Mental Science. 184:503-508.

21. Drake M T, Clarke B L, Lewiecki E M. 2015. The Pathophysiology and Treatment of Osteoporosis. Clinical Therapeutics. 37(8):1837-1850.

22. Snyder PJ, Kopperdahl DL, Stephens-Shields AJ, Ellenberg SS, Cauley JA, Ensrud KE, Lewis CE, BarrettConnor E, Schwartz AV, Lee DC, Bhasin S, Cunningham GR, Gill TM, Matsumoto AM, Swerdloff RS, Basaria S, Diem SJ, Wang C, Hou X, Cifelli D, Dougar D, Zeldow B, Bauer DC, Keaveny TM. 2017. Effect of Testosterone Treatment on Volumetric Bone Density and Strength in Older Men With Low Testosterone: A Controlled Clinical Trial. JAMA Internal Medicine. 1;177(4):471-479.

23. Tseng PT, Chen YW, Yeh PY, Tu KY, Cheng YS, Wu CK. 2015. Bone Mineral Density in Schizophrenia: An Update of Current Meta-Analysis and Literature Review Under Guideline of PRISMA. Medicine;94(47):e1967.

24. Lally J, Sahl AB, Murphy KC, Gaughran F, Stubbs B. 2019. Serum Prolactin and Bone Mineral Density in Schizophrenia: A Systematic Review. Clinical Psychopharmacology And Neuroscience: The Official Scientific Journal Of The Korean College Of Neuropsychopharmacology. 17(3):333-342.

25. Markianos M, Hatzimanolis J, Lykouras L. 2001. Neuroendocrine responsivities of the pituitary dopamine system in male schizophrenic patients during treatment with clozapine, olanzapine, risperidone, sulpiride, or haloperidol. European Archives Of Psychiatry And Clinical Neuroscience. 251(3):141-146.

26. Liang Y, Huang J, Tian JB, Cao YY, Zhang GL, Wang CG, Cao Y, Li JR. 2016. Factors associated with decreased bone mineral density in postmenopausal women with schizophrenia. Clinical Interventions In Aging. 11:153-157.

27. Doumouchtsis KK, Kostakis AI, Doumouchtsis SK, Grapsa EI, Passalidou IA, Tziamalis MP, Poulakou MV, Vlachos IS, Perrea DN. 2008. The effect of sex hormone abnormalities on proximal femur bone mineral density in hemodialysis patients and the possible role of RANKL. Hemodialysis International. 12(1):100107.

28. Prior JC. 2018. Progesterone for the prevention and treatment of osteoporosis in women. Climacteric. 21(4):366-374.

29. Aydin T, Karacan I, Demir SE, Sahin Z. 2005. Bone loss in males with ankylosing spondylitis: its relation to sex hormone levels. Clinical Endocrinology. 63(4):467-469. 
339

340

341

342

343

344

345

346

347

348

349

350

351

352

353

354

30. Seven A, Yuksel B, Kabil Kucur S, Yavuz G, Polat M, Unlu BS, Keskin N. 2016. The evaluation of hormonal and psychological parameters that affect bone mineral density in postmenopausal women. European Review For Medical And Pharmacological Sciences. 20(1):20-25.

31. Kinon BJ, Liu-Seifert H, Stauffer VL, Jacob J. 2013. Bone loss associated with hyperprolactinemia in patients with schizophrenia. Clinical Schizophrenia \& Related Psychoses. 7(3):115-123.

32. Jung DU, Conley RR, Kelly DL, Kim DW, Yoon SH, Jang JH, Shin JG, Shim JC. 2006. Prevalence of bone mineral density loss in Korean patients with schizophrenia: a cross-sectional study. The Journal Of Clinical Psychiatry. 67(9):1391-1396.

33. Doknic M, Maric NP, Britvic D, Pekic S, Damjanovic A, Miljic D, Stojanovic M, Radojicic Z, Jasovic Gasic M, Popovic V. 2011. Bone remodeling, bone mass, and weight gain in patients with stabilized schizophrenia in real-life conditions treated with long-acting injectable risperidone. Neuroendocrinology. 94(3):246-254.

34. Bulut SD, Bulut S, Atalan DG, Tulaci RG, Türker T, Gürçay E, Aydemir Ç. 2016. The Effect of Antipsychotics on Bone Mineral Density and Sex Hormones in Male Patients with schizophrenia. Psychiatria Danubina.28(3):255-262. 


\section{Table $\mathbf{1}$ (on next page)}

Comparison between schizophrenia patients and control subjects

BMI: body mass index, BMD: bone mass density 
1 Table 1 Socio-demographic and clinical data of the study subjects.

\begin{tabular}{lllll}
\hline & Patients $(\mathrm{N}=250)$ & Controls $(\mathrm{N}=288)$ & $\mathrm{t} / \mathrm{Z} / \mathrm{X}^{2}$ & $\mathrm{P}$ \\
\hline Age (years) & $46.3 \pm 10.6$ & $46.0 \pm 10.8$ & -0.51 & 0.613 \\
Sex (male/female) & & & & 0.101 \\
$\quad$ Male & 137 & 137 & & \\
Female & 113 & 151 & & \\
Body mass index $\left(\mathrm{Kg} / \mathrm{m}^{2}\right)$ & $24.5 \pm 4.0$ & $23.4 \pm 3.5$ & -2.704 & 0.007 \\
Total disease courses $($ years $)$ & $21.0 \pm 9.0$ & - & & \\
Bone mass density $\left(\mathrm{g} / \mathrm{cm}^{2}\right)$ & $0.5 \pm 0.1$ & $0.5 \pm 0.1$ & -3.60 & $<0.001$ \\
Osteoporosis & & & & $<0.001$ \\
Yes & 61 & 29 & & \\
No & 189 & 259 & & \\
\hline
\end{tabular}

2

3

4

5

6

7

8

9

10

11

12

13

14

15

16

17

18

19

20

21

22

23

24

25 


\section{Table 2 (on next page)}

Differences between schizophrenia patients with osteoporosis and without osteoporosis

BMI: body mass index, BMD: bone mass density, E2: Estradiol , T: Testosterone, P:

Progesterone, PRL: prolactin , FSH: Follicle-stimulating hormone, LH: luteinizing hormone. 
1 Table 2 Differences between schizophrenia patients with osteoporosis and without osteoporosis.

\begin{tabular}{lcccl}
\hline & $\begin{array}{c}\text { Patients with } \\
\text { osteoporosis }\end{array}$ & $\begin{array}{c}\text { Patients without } \\
\text { osteoporosis }\end{array}$ & $\mathrm{t} / \mathrm{Z} / \mathrm{X}^{2}$ & $\mathrm{P}$ \\
\cline { 2 - 4 } & $\mathrm{N}=61$ & $\mathrm{~N}=189$ & & \\
\hline Age (year) & $51.7 \pm 11.6$ & $44.6 \pm 9.7$ & -4.6 & $<0.001$ \\
Sex (male/female) & $40 / 21$ & $97 / 92$ & & 0.052 \\
Course of disease (years) & $26.2 \pm 9.3$ & $19.5 \pm 8.8$ & -4.9 & $<0.001$ \\
Body mass index (kg/m ${ }^{2}$ ) & $23.1 \pm 3.1$ & $24.9 \pm 4.1$ & 3.5 & 0.001 \\
Daily dosage of risperidone & $4.8 \pm 1.7$ & $4.7 \pm 1.9$ & -0.7 & 0.466 \\
Estradiol (pmol/L) & $55.3 \pm 24.6$ & $88.0 \pm 45.3$ & -5.3 & $<0.001$ \\
Testosterone (nmol/L) & $9.2 \pm 5.5$ & $8.9 \pm 6.3$ & -0.3 & 0.730 \\
Progesterone (nmol/L) & $0.6 \pm 0.3$ & $0.7 \pm 0.5$ & -1.0 & 0.340 \\
Prolactin (ug/L) & $90.9 \pm 20.5$ & $44.1 \pm 17.2$ & -10.6 & $<0.001$ \\
Follicle-stimulating hormone (IU/L) & $7.6 \pm 4.1$ & $6.6 \pm 4.0$ & -2.2 & 0.026 \\
Luteinizing hormone (IU/L) & $9.0 \pm 3.5$ & $8.8 \pm 4.4$ & -1.0 & 0.302 \\
\hline
\end{tabular}




\section{Table 3(on next page)}

Results of the stepwise logistic regression analysis: independent risk factors for osteoporosis in schizophrenia patients

E2: Estradiol , PRL: prolactin 
1 Table 3. Results of binary logistic regression analysis: factors associated with osteoporosis in 2 schizophrenia patients.

\begin{tabular}{lllcc}
3 & Predictors & Sig & Odds ratio & 95\%CI for Exp.(B) \\
\cline { 2 - 5 } 5 & Age(years) & 0.773 & 0.9 & $0.85 \sim 1.13$ \\
6 & Total disease courses(years) & 0.176 & 1.1 & $0.95 \sim 1.34$ \\
7 & Bone mass density $\left(\mathrm{Kg} / \mathrm{m}^{2}\right)$ & 0.826 & 1.0 & $0.86 \sim 1.13$ \\
8 & Estradiol (pmol/L) & 0.011 & 0.9 & $0.96 \sim 1.00$ \\
9 & Prolactin (ug/L) & 0.000 & 1.1 & $1.09 \sim 1.15$ \\
& Follicle-stimulating hormone, (IU/L) & 0.407 & 1.1 & $0.92 \sim 1.22$ \\
& Daily dosage of risperidone & 0.407 & 1.1 & $0.66 \sim 1.19$ \\
\hline
\end{tabular}

Total model: $P$ $<0.001$, $\mathrm{R}$ square: 0.5 20 